EPJ Web of Conferences 57, 03006 (2013)

DOI: $10.1051 /$ epjconf/20135703006

(C) Owned by the authors, published by EDP Sciences, 2013

\title{
Hybrid atom-membrane optomechanics
}

\author{
Maria Korppi ${ }^{1}$, Andreas Jöckel ${ }^{1}$, Matthew T. Rakher ${ }^{1}$, Stephan Camerer ${ }^{2,3}$, \\ David Hunger ${ }^{2,3}$, Theodor W. Hänsch ${ }^{2,3}$ and Philipp Treutlein ${ }^{1, a}$ \\ ${ }^{1}$ Departement Physik, Universität Basel, Klingelbergstrasse 82, 4056 Basel, Switzerland \\ ${ }^{2}$ Fakultät für Physik, Ludwig-Maximilians-Universität, Schellingstraße 4, 80799 München, \\ Germany \\ ${ }^{3}$ Max-Planck-Institut für Quantenoptik, Hans-Kopfermann-Str. 1, 85748 Garching, Germany
}

\begin{abstract}
We report on the realization of a hybrid optomechanical system in which ultracold atoms are coupled to a micromechanical membrane. The atoms are trapped in the intensity maxima of an optical standing wave formed by retroreflection of a laser beam from the membrane surface. Vibrations of the membrane displace the standing wave, thus coupling to the center-of-mass motion of the atomic ensemble. Conversely, atoms imprint their motion onto the laser light, thereby modulating the radiation pressure force on the membrane. In this way, the laser light mediates a long-distance coherent coupling between the two systems. When the trap frequency of the atoms is matched to the membrane frequency, we observe resonant energy transfer. Moreover, we demonstrate sympathetic damping of the membrane motion by coupling it to laser-cooled atoms. Theoretical investigations show that the coupling strength can be considerably enhanced by placing the membrane inside an optical cavity. This could lead to quantum coherent coupling and ground-state cooling of the membrane via a distant atomic ensemble.
\end{abstract}

\section{INTRODUCTION}

Laser light can exert a mechanical force on material objects through radiation pressure and through the optical dipole force $[1,2]$. These forces have been used for decades to achieve extraordinary control over the quantum states of atoms, molecules, and ions. Recently, solid-state physicists have started to achieve similar control over individual vibrational modes of high-quality fabricated mechanical structures. In the very active field of optomechanics [3-7], light forces are exploited for cooling and control of the vibrations of mechanical oscillators ranging from macroscopic mirrors to micromembranes and nanoscale cantilevers. Notably, the ground-state of a single mechanical mode of an optomechanical crystal was reached by laser-cooling [8]. Experiments with other types of mechanical oscillators have also reached phonon occupation numbers very close to the ground state [9-11]. The goal is to ultimately control these devices on the quantum level, analogously to what can be achieved with atomic systems. This would allow one to study quantum physics on a macroscopic scale [7], possibly revealing yet unobserved quantum decoherence mechanisms [12, 13]. In addition, such devices could provide quantum-limited force-sensing in precision measurements [14].

Combining the aforementioned advancements in atomic and solid-state physics, a number of recent theoretical articles have proposed that light forces could be used to couple the motion of atoms in a trap to the vibrations of a single mode of a mechanical oscillator [15-24]. In the resulting hybrid optomechanical system, the well-established toolbox of atomic physics could be used to control the

\footnotetext{
ae-mail: philipp.treutlein@unibas.ch
}

This is an Open Access article distributed under the terms of the Creative Commons Attribution License 2.0, which permits unrestricted use, distribution, and reproduction in any medium, provided the original work is properly cited. 


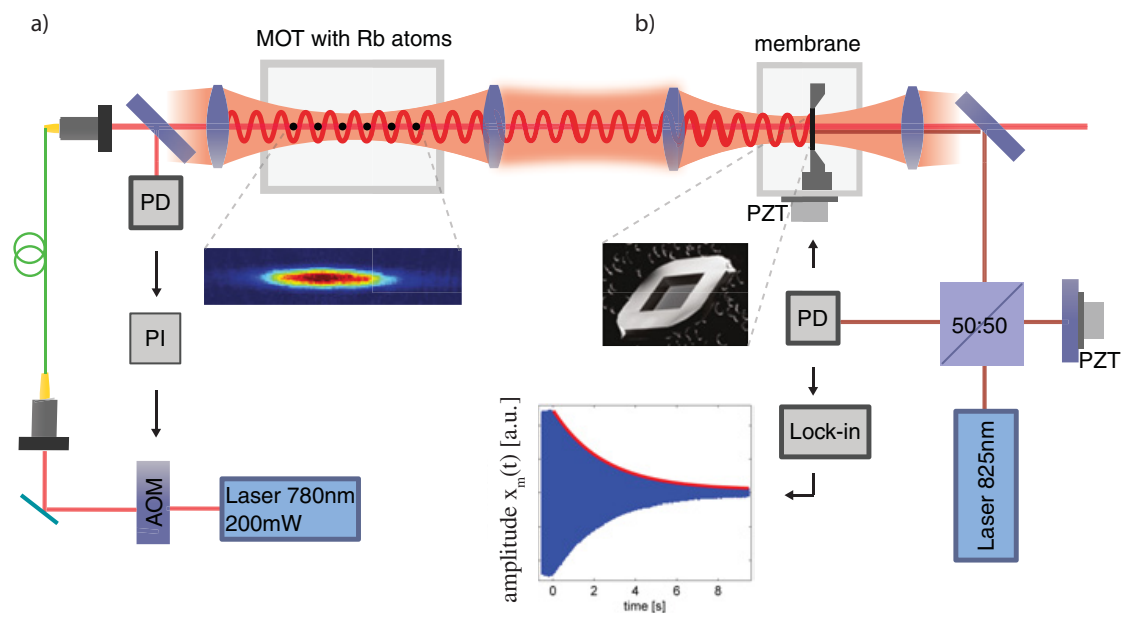

Figure 1. Experimental setup. a) The optical lattice: ${ }^{87} \mathrm{Rb}$ atoms are collected in a magneto-optical trap (MOT) inside an ultra-high vacuum chamber. The lattice laser $(780 \mathrm{~nm})$ is fiber coupled, power stabilized with a PI regulator and an acousto-optic modulator (AOM), and focused into the MOT vacuum chamber. b) The membrane resides in a second room-temperature vacuum chamber and serves as partially reflective end mirror for the 1D optical lattice. The membrane motion is read out with a Michelson interferometer (laser wavelength $825 \mathrm{~nm}$ ). The interferometer signal from the photodetector (PD) is frequency-split: the low-frequency part is used for interferometer stabilization via the piezo-mirror in one of the interferometer arms (PZT); the high-frequency part including the membrane signal is used for readout and piezo (PZT) feedback drive of the membrane. The membrane amplitude is measured with a lock-in amplifier and an oscilloscope. The inset shows a measured ringdown of the membrane.

vibrations of an engineered mechanical device. Atoms could be used to read out the motion of the oscillator, to manipulate its dissipation, and ultimately to perform quantum information tasks such as coherently exchanging the quantum state of the two systems. Moreover, the oscillator could serve as a new tool in atomic physics experiments, for example as a transducer coupling different types of atomic and molecular degrees of freedom. In pioneering experiments, atoms were used to detect vibrations of micromechanical oscillators using magnetic [25] or surface-force coupling [26]. However, the backaction of the atoms onto the oscillator's motion, which is required for cooling and manipulating the oscillator with the atoms, could not yet be observed.

In the hybrid optomechanical system described here, a cloud of ultracold atoms is coupled via laser light to a microscopic membrane oscillator. In a proof-of-principle experiment, we have observed the backaction of the atoms onto the membrane oscillator, as reported in Ref. [27]. The present article reviews these experiments, including a more detailed theoretical description of our system and a discussion of the conditions required to observe normal mode splitting. In addition, we provide an outlook where we address significant improvements that are currently being implemented in a new setup to further enhance the coupling. With these advancements, ground state cooling of the oscillator seems feasible.

\section{DESCRIPTION OF THE EXPERIMENTAL SETUP}

A unique feature of our system is the long-distance coupling provided by the optical lattice, allowing us to place the membrane and the atoms into separate vacuum chambers as shown in Fig. 1. A laser beam traverses through the atomic chamber, is retroreflected at the membrane, and forms an optical lattice for the atoms. The modularity of the setup allows us to change the mechanical oscillators relatively quickly without affecting the cold atom preparation in the ultra-high vacuum (UHV) chamber. Furthermore, the 
membrane could be placed inside a cryostat without adding complexity to the atomic side. In the future one could think of adding additional modules to the setup to built up a more complex network of coupled (quantum) systems. Next, we discuss the membrane and the atomic modules separately.

\subsection{SiN membrane}

Silicon nitride $(\mathrm{SiN})$ membranes have garnered a great deal of interest in optomechanics experiments owing to their extraordinary mechanical and optical properties [28-32]. The SiN membrane used in our experiment has dimensions of $0.5 \mathrm{~mm} \times 0.5 \mathrm{~mm} \times 50 \mathrm{~nm}$ and a tensile stress of about $120 \mathrm{MPa}$ [28]. Its fundamental vibrational mode has a measured frequency of $\omega_{m} / 2 \pi=272 \mathrm{kHz}$ and an effective mass of $M=1 \times 10^{-11} \mathrm{~kg}$. The power reflectivity of the membrane is $\mathfrak{r}=0.28$ at $\lambda=780 \mathrm{~nm}$. The membrane is kept at room temperature at a pressure of $10^{-6}$ mbar. The membrane motion is measured with a Michelson interferometer, as illustrated in Fig. 1b, where the membrane itself terminates one arm of the interferometer. The reference arm-length is actively stabilized with a mirror that is attached to a piezo. The power in the interferometer is $3 \mathrm{~mW}$. The interferometer has a displacement sensitivity of $3 \times 10^{-14} \mathrm{~m} / \sqrt{\mathrm{Hz}}$, which can resolve the thermal amplitude of the membrane with signal to noise ratio of 1000 in a $1 \mathrm{~Hz}$ bandwidth. The mechanical quality factor $Q=\omega_{m} / \gamma_{m}=\omega_{m} \tau / 2$ was determined in ringdown measurements from the $1 / e$ decay time $\tau$ of the membrane amplitude with a lock-in technique (a ringdown measurement is presented in the inset in Fig. 1b). We observe a quality factor of $Q=8.5 \times 10^{5}$ for the fundamental mechanical mode.

When the lattice laser beam illuminates the membrane, the finite absorption of the membrane results in local heating. This leads to thermal expansion and consequently reduced tensile stress [30]. Thus, we observe a deterministic decrease of $\omega_{m}$ with increasing lattice laser power. We also observe that the mechanical $Q$ changes in a repeatable but non-monotonic way as function of $\omega_{m}$ due to the coupling of the membrane to its support modes of same frequency, as analyzed in detail in Ref. [30]. For example, when the laser power is $P=76 \mathrm{~mW}$, we measure $Q=1.5 \times 10^{6}$ in contrast to the value of $Q=8.5 \times 10^{5}$ given above, which was measured when the lattice laser was off. These changes due to absorption are accounted for in the subsequent experimental analysis by doing a reference measurement where the lattice laser is kept on but no atoms are trapped in the lattice.

\subsection{Optical lattice}

The techniques to prepare, manipulate, and readout the motional and internal states of ultracold atoms on the quantum level are well-established. Combined with long ground state coherence times, this makes ultracold atoms a very attractive quantum system. Here we couple the membrane vibrations to the center of mass (c.o.m.) motion of atoms in an optical lattice. In our setup, the atoms are kept in a ultra high vacuum (UHV) environment as illustrated in Fig. 1a and laser-cooled to a temperature of $100 \mu \mathrm{K}$ in a magneto optical trap (MOT) [33]. The optical lattice potential is provided by a grating-stabilized diode laser that is injected into a tapered amplifier to obtain a power level of up to $140 \mathrm{~mW}$ at the position of the atoms. The output of the laser is linearly polarized and its power $P$ is actively stabilized using an acousto-optic modulator (AOM) to a relative stability of $2 \times 10^{-4}$ r.m.s. in a bandwidth of $12 \mathrm{kHz}$. The laser frequency is red-detuned by $\Delta_{\text {opt }}=-2 \pi \times 21 \mathrm{GHz}$ from the $D_{2}$ line of ${ }^{87} \mathrm{Rb}\left(F=2 \leftrightarrow F^{\prime}=3\right.$ transition). The laser beam is focused into the atomic chamber with a beam waist $w_{0}=350 \mu \mathrm{m}$ at the position of the atoms and is partially reflected at the surface of a SiN membrane mounted in a separate vacuum chamber. The reflected beam is overlapped with the incoming beam such that a $1 \mathrm{D}$ optical lattice potential for the ultracold atoms is generated [34].

The optical lattice provides a sinusoidal potential for the atoms of the form [35]

$$
V(x)=V_{0} \sin ^{2}(k x)+V_{\text {offset }}
$$


a) membrane -> atoms

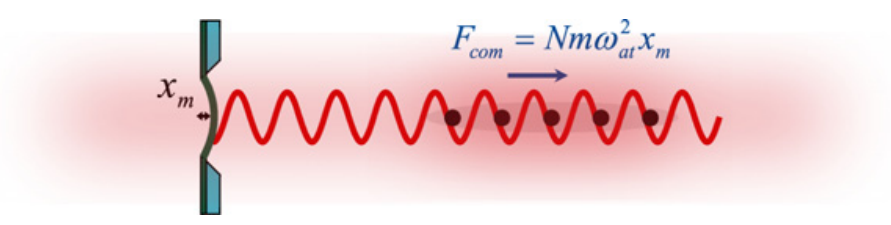

b) atoms -> membrane (back-action)

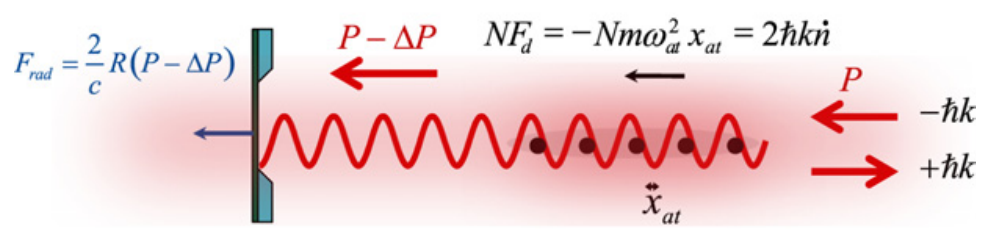

Figure 2. a) Illustration of the action of the membrane onto the atoms. b) The atoms act back onto the membrane via redistribution of photons between the two counter-propagating laser beams, which causes a power modulation $\Delta \mathrm{P}$ of the light that hits the membrane.

where $k=2 \pi / \lambda$ is the wave-vector of the lattice laser. The constant offset $V_{\text {offset }}$ arises because the lattice potential is not fully modulated. This is due to the finite reflectivity $\mathfrak{r}=0.28$ of the membrane and the finite transmission of the optical elements between the membrane and the atoms, $\mathfrak{t}=0.82$. In a harmonic approximation at the bottom of the potential wells, the trap frequency of the atoms along the lattice direction is given by $\omega_{a t}=\sqrt{2 V_{0} k^{2} / m}$, where $m$ is the atomic mass. The lattice depth, $V_{0}$, scales as $V_{0} \propto P /\left(\Delta_{\mathrm{opt}} w_{0}^{2}\right)$. Thus, by changing the power of the lattice beam while keeping $\Delta_{\mathrm{opt}}$ and $w_{0}$ fixed, we can tune the trap frequency of the atoms to match that of the membrane. Under this condition the coupling can lead to resonant energy transfer between the atoms and the membrane as described in Sec. 3 below. When the power is tuned to $P=76 \mathrm{~mW}$ such that $\omega_{a t} \approx \omega_{m}$, the calculated modulation depth of the sinusoidal potential is $V_{0}=k_{B} \times 290 \pm 50 \mu \mathrm{K}$ and $\omega_{a t} / 2 \pi=305 \pm 25 \mathrm{kHz}$ [27]. At $P=76 \mathrm{~mW}$, we typically load $N=2 \times 10^{6}$ atoms into the lattice with a temperature of $T=100 \mu \mathrm{K}$ as determined by absorption imaging.

\section{COUPLING MECHANISM}

\subsection{Semiclassical model}

In our system, the membrane-light coupling is due to radiation pressure and the atom-light coupling is due to the optical dipole force. To understand this coupling, let us start by investigating the effect of the membrane onto the atoms, which is illustrated in Figure 2a. A displacement of the membrane, $x_{m}$, displaces the lattice potential, resulting in a dipole force $F=m \omega_{a t}^{2} x_{m}$ onto each atom, where $m$ is the atomic mass. The membrane motion thus couples through $F_{c o m}=N F$ to the center of mass (c.o.m.)motion of an ensemble of $N$ atoms trapped in the lattice. An oscillating membrane will parametrically excite the atoms that have a trap frequency equal to that of the membrane. In the experiment we can apply additional laser cooling to the atoms to keep the c.o.m. motion of the atoms in steady state.

Vice versa, the atoms will imprint a signature of their c.o.m. motion onto the light field. An atom displaced by $x_{a t}$ from the bottom of its potential well experiences a restoring optical dipole force $F_{d}=-m \omega_{a t}^{2} x_{a t}$ from the lattice. This is illustrated in Figure $2 \mathrm{~b}$. On a microscopic level, $F_{d}$ arises from absorption followed by stimulated emission, leading to a redistribution of photons between the two running wave components forming the lattice $[36,37]$ : the displaced atom preferentially absorbs 
photons from one of the lattice beams and reemits them into the other. Each redistribution event results in a momentum transfer of $\pm 2 \hbar k$ to the atom. Consequently, the photon redistribution modulates the power of the laser beam traveling towards the membrane by $\Delta P=\hbar \omega \dot{n}=-\frac{c}{2} N F_{d}$, where $\dot{n}$ is the total photon redistribution rate due to the $N$ atoms. This leads to a modulation of the radiation pressure force acting on the membrane $\Delta F_{\text {rad }}=\frac{2}{c} \mathfrak{r t} \Delta P=-\mathfrak{r t} N F_{d}=\mathfrak{r t} N m \omega_{a t}^{2} x_{a t}$. Some of the photons that have interacted with the atoms are lost because $\mathfrak{r t}<1$ and do not contribute to the force on the membrane. If losses in the beam path and finite membrane reflectivity are neglected $(\mathfrak{r t}=1)$, the forces experienced by the displaced atoms and the membrane are exactly equal in magnitude, but opposite in sign, following the action-reaction principle. However, in our experimental realization the coupling is asymmetric.

\subsection{Coupled oscillator dynamics}

In a simple model of damped harmonic oscillators coupled through $F_{c o m}$ and $\Delta F_{\text {rad }}$, the equations of motion for the fundamental vibrational mode of the membrane and the c.o.m. motion of the atoms can be written as

$$
\begin{aligned}
& \dot{p}_{a t}=-\gamma_{a t} p_{a t}-N m \omega_{a t}^{2} x_{a t}+N m \omega_{a t}^{2} x_{m} \\
& \dot{x}_{a t}=p_{a t} / N m \\
& \dot{p}_{m}=-\gamma_{m} p_{m}-M \omega_{m}^{2} x_{m}+\mathfrak{r t} N m \omega_{a t}^{2} x_{a t}, \\
& \dot{x}_{m}=p_{m} / M
\end{aligned}
$$

where $\gamma_{m}\left(\gamma_{a t}\right)$ is the motional damping rate of the membrane (atoms). We introduce dimensionless complex amplitudes $\quad a=e^{i \omega_{m} t} \sqrt{N m \omega_{a t} / 2 \hbar}\left(x_{a t}+i p_{a t} / N m \omega_{a t}\right) \quad$ and $\quad b=e^{i \omega_{m} t} \sqrt{M \omega_{m} / 2 \hbar}$ $\left(x_{m}+i p_{m} / M \omega_{m}\right)$ in a frame rotating at $\omega_{m}$. In a classical description, $a$ and $b$ are the amplitudes of the two oscillators in phase space; in a quantum description they are promoted to annihilation operators. We make the rotating-wave approximation (RWA), i.e. we neglect fast rotating terms $\propto e^{2 i \omega_{m} t}$ in the resulting equations of motion for $a$ and $b$. This is justified since we consider the regime where $\omega_{m} \approx \omega_{a t} \gg g, \gamma_{a t}, \gamma_{m}$. This results in a set of coupled equations:

$$
\begin{aligned}
& \dot{a}=-i \delta a-\left(\gamma_{a t} / 2\right) a+i g b \\
& \dot{b}=-\left(\gamma_{m} / 2\right) b+i \operatorname{rtg} a,
\end{aligned}
$$

where $\delta=\omega_{a t}-\omega_{m}$ is the atom-membrane detuning. We have defined the coupling constant as $g=\frac{\omega_{a t}}{2} \sqrt{\frac{N m \omega_{a t}}{M \omega_{m}}}$. Even though the mass ratio of an atom to the membrane will be exceedingly small under reasonable conditions $\left(m / M \approx 10^{-14}\right)$, the coupling can still be significant due to collective enhancement by the large number of atoms.

The coupling leads to both a modified damping rate (dispersive part of the coupling) as well as a frequency shift (reactive part). Both effects are obtained by solving for the eigenvalues $v_{ \pm}$of Eqs. (3):

$$
v_{ \pm}=-\frac{\gamma_{a t}+\gamma_{m}+2 i \delta}{4} \pm \sqrt{\left(\frac{\gamma_{a t}-\gamma_{m}+2 i \delta}{4}\right)^{2}-\mathfrak{r t g} g^{2}}
$$

The normal mode oscillation frequencies are given by $\operatorname{Im}\left\{v_{ \pm}\right\}$, while the amplitude damping rates are given by $\mathfrak{R e}\left\{v_{ \pm}\right\}$. In our experiment, we operate in the weak-coupling regime where $\gamma_{a t} \gg g, \gamma_{m}$. In this regime, the frequency shift due to the coupling is zero on resonance within the RWA. A calculation beyond the RWA shows that the frequency shift is $\ll 1 \mathrm{~Hz}$ for our parameters, and thus not observable in the experiment. On the other hand, in the strong-coupling regime where $g \gg\left(\gamma_{a t}, \gamma_{m}\right)$, the coupling leads to a normal-mode splitting of $2 g \sqrt{\mathfrak{r t}}$ on resonance. 
In the weak-coupling regime $\left(\gamma_{a t} \gg g, \gamma_{m}\right)$, the energy damping rate of the membrane, $-2 \Re \mathfrak{R}\left\{v_{+}\right\}$, is given to lowest order in $g$ by

$$
\Gamma=\gamma_{m}+\gamma_{a t} \frac{g^{2} \mathfrak{r t}}{\delta^{2}+\left(\gamma_{a t} / 2\right)^{2}} .
$$

The second term in Eq. (5) is the additional dissipation rate of the membrane motion due to coupling to atoms,

$$
\Delta \gamma=\Gamma-\gamma_{m}=\gamma_{a t} \frac{g^{2} \mathfrak{r t}}{\delta^{2}+\left(\gamma_{a t} / 2\right)^{2}} \propto N .
$$

In our experimental realization, we have two different contributions to the overall atomic damping rate

$$
\gamma_{a t}=\gamma_{c}+\gamma_{\phi}
$$

The first, $\gamma_{c}$, is the laser cooling rate due to the MOT. The second, $\gamma_{\phi}$, describes additional dephasing of the c.o.m. motion due to finite temperature of the atoms and the spatial dependence of the trap frequency on the lattice laser intensity profile. The value of $\gamma_{\phi}$ is intrinsic to the experimental realization and constant when the atomic motion is in steady-state. On the other hand, the laser cooling rate $\gamma_{c}$ can be adjusted. In our experiment, we apply strong laser cooling to the atoms, $\gamma_{c} \gg g, \gamma_{m}$, so that the atomic c.o.m. amplitude is approximately in steady state $(\dot{a} \simeq 0)$ on the much slower timescale of membrane dynamics. In this way we can dissipate energy from the coupled system, resulting in sympathetic damping of the membrane vibrations via the atoms as described by Eq. (6). We have perfomed two different measurements to characterize this effect. First, we measured $\Delta \gamma(\delta)$ across the resonance, and second, we tuned the system on resonance, $\delta \approx 0$, and investigated the scaling of $\Delta \gamma$ with respect to $N$. The results are presented in Section 4.

\subsection{Note on quantum dynamics}

The foregoing considerations did not provide any information about the noise processes that fundamentally limit the performance of our system. A fully quantum treatment of our system has been described in Ref. [23]. The theory shows that the results of the simple model described above hold for the corresponding quantum-mechanical expectation values in the absence of noise processes. ${ }^{1}$ In addition, the full theory derives various noise sources, such as radiation pressure noise acting on the membrane, thermal heating of the membrane due to coupling to its support and absorption of laser light, and the momentum diffusion of the atoms due to spontaneous emission. The momentum diffusion of the atoms can be suppressed by preparing the atoms in the ground state of the lattice potential, as experimentally demonstrated e.g. in Ref. [38]. On the membrane side, thermal heating is the dominant noise process, which is described by the coupling of the membrane to a thermal bath with mean phonon occupation number $n_{t h} \simeq k_{B} T / \hbar \omega_{m}$. In the presence of these imperfections and for $\gamma_{a t} \gg g, \gamma_{m}$, the steady-state phonon occupation of the membrane is [23]

$$
\bar{n}=\frac{\gamma_{m}}{\Gamma} n_{t h}+\left(\frac{\gamma_{a t}}{4 \omega_{m}}\right)^{2} .
$$

Ground-state cooling of the membrane via the atoms is possible for $\Gamma \gg \gamma_{m} n_{t h}$ and $\gamma_{a t} \ll \omega_{m}$. To achieve a sufficiently small $n_{t h}$, cryogenic precooling of membrane is required [23].

\footnotetext{
1 Note a difference in notation: here and in Ref. [27] we define the coupling constant as $g=\frac{\omega_{a t}}{2} \sqrt{\frac{N m \omega_{a t}}{M \omega_{m}}}$, whereas in Ref. [23] it is defined as $g=\omega_{a t} \sqrt{\frac{N m \omega_{a t}}{M \omega_{m}}}$. Note also that there is a typo in the effective cooling rate on resonance given in Ref. [23], it should $\operatorname{read} \Gamma_{m}=\gamma_{m}+\mathfrak{r} g^{2} / \gamma_{a t}^{c o o l}$ using the notation of that paper.
} 


\section{ICAP 2012}

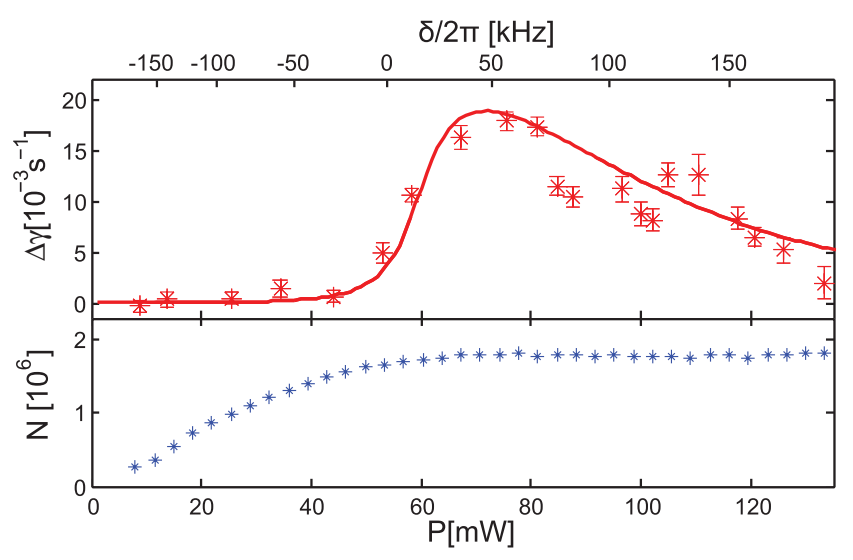

Figure 3. Backaction of laser-cooled atoms onto the membrane. Top: measured additional membrane dissipation rate $\Delta \gamma=\Gamma-\gamma_{m}$ due to coupling to atoms as a function of $P$. The rates $\Gamma$ and $\gamma_{m}$ are extracted from exponential fits to averaged decay curves $(2 \times 455$ experimental runs per datapoint $)$. Solid line: theory for a thermal ensemble in the lattice. Bottom: atom number $N$ in the lattice measured by absorption imaging.

Our system bears some analogies with cavity-optomechanics, where a mechanical oscillator is coupled to an optical cavity via radiation pressure forces [3-7]. Comparing Eq. (8) with the corresponding equation for cavity-optomechanical cooling [39], we find that our atomic damping rate $\gamma_{a t}$ corresponds to the cavity decay rate $\kappa$ in the resolved-sideband regime. Notably, in contrast to the usual cavity-optomechanical setup where $\kappa$ is a fixed parameter, in our setup $\gamma_{a t}$ is tunable via $\gamma_{c}$. Thus, for sufficiently strong $g$ and large $\gamma_{a t}>g$, sympathetic ground-state cooling of the membrane should be feasible via efficient laser-cooling of the atoms [23]. Having reached the ground-state, one could conveniently switch off the cooling and study the system evolution in the regime of strong coherent coupling, where the coupling rate $g$ exceeds all the decoherence rates.

\section{EXPERIMENTAL RESULTS: OPTOMECHANICAL DAMPING OF THE MEMBRANE}

The backaction of the laser-cooled atomic ensemble onto the membrane vibrations is observed in membrane ringdown measurements. While the lattice is continuously loaded from the MOT (Sec. 2.2), the membrane is resonantly excited to an amplitude of $540 \mathrm{pm}$. After switching the excitation off, the decay of the membrane amplitude is recorded. We performed alternating experiments with and without atoms in the lattice, where the presence of atoms was controlled by detuning the MOT laser frequency, but the laser power on the membrane was kept fixed. This type of reference measurement allows straighforward determination of the change in the decay rate $\Delta \gamma$ as predicted by Eq. (6), without being perturbed by the change in the $Q$-factor of the membrane with laser power (see Sec. 2.1).

The measured $\Delta \gamma$ is shown in Fig. 3 as a function of $P$ and $\delta$. Even though the number of atoms in the lattice changes with $P$, near the resonance it does not change significantly as shown in the lower part of Fig. 3. We observe a broad resonance in $\Delta \gamma$ around $P \approx 76 \mathrm{~mW}$. The resonance is broadened and shifted to $\delta>0$. This can be explained with a more elaborate theory that includes the finite temperature of the atoms and the lattice anharmonicity, which results in the solid curve shown in red in Fig. 3 (further details in Sec. 4).

In a second experiment, we measured and verified the scaling of $\Delta \gamma$ with atom number $N$ (see Eq. (6)). We prepared the system on resonance $(P=76 \mathrm{~mW})$ and varied the number of atoms that are loaded into the lattice by varying the power of the MOT repump laser. We observe a linear dependence 


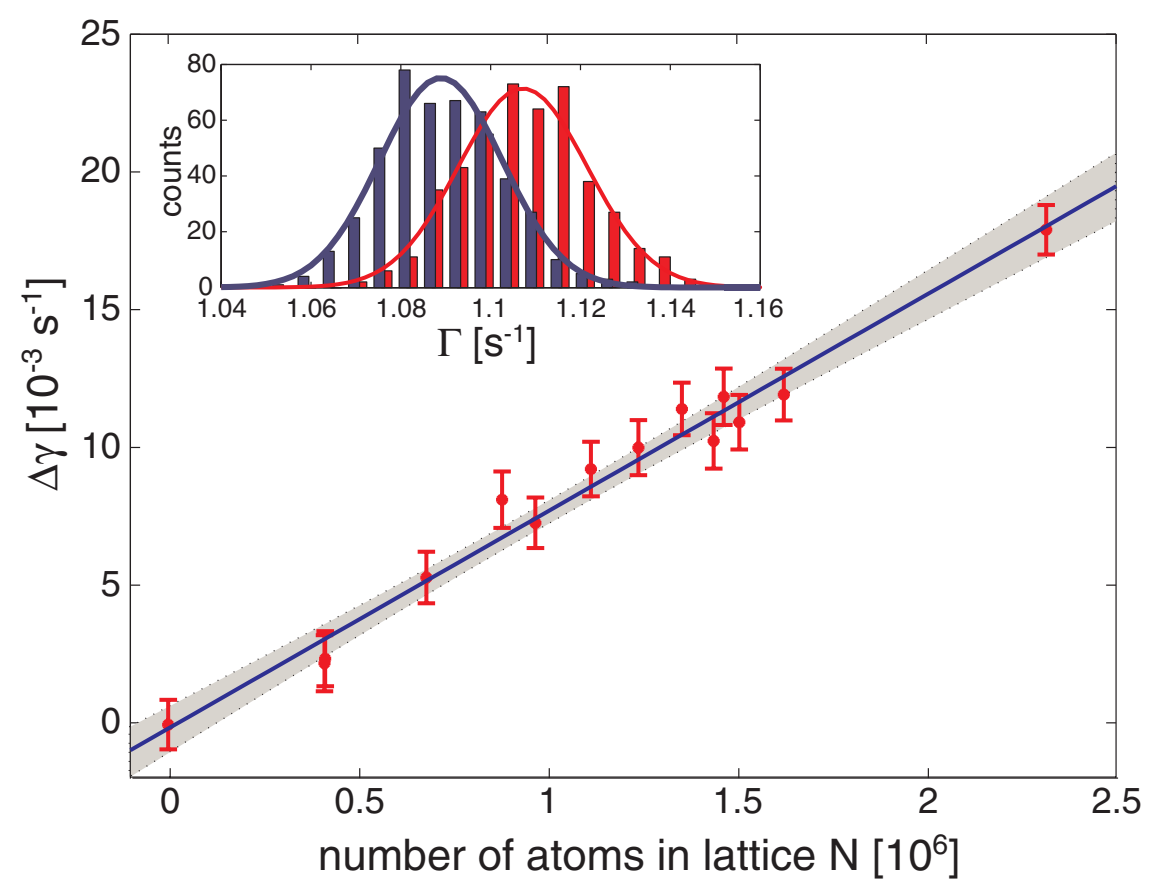

Figure 4. Measured additional membrane dissipation $\Delta \gamma$ as a function of atom number $N$ for resonant coupling $(P=76 \mathrm{~mW})$. The blue line is a linear fit. The observed dependence agrees well with theory. Inset: histogram of measurements of $\Gamma$ for $N=2.3 \times 10^{6}$ (red) and $N=0$ (blue).

of $\Delta \gamma$ on $N$ as shown in Fig. 4, in agreement with Eq. (6) as well as with the theory in Ref. [23]. The high $Q$ of the membrane allows us to precisely measure minute changes in $\Delta \gamma$.

In order to compare our measurements with theory, we calculate $\Delta \gamma$ from Eq. (6). The overall atomic damping rate is taken as the full-width-half-maximum of the resonance in Fig. 3, $\gamma_{a t}=$ $2 \pi \times 130 \pm 26 \mathrm{kHz}$, and the atom number is measured to be $N=(2.3 \pm 0.5) \times 10^{6}$. For these values, the theory predicts a value of $\Delta \gamma=0.023 \pm 0.005 \mathrm{~s}^{-1}$ for $\delta=0$, whereas the measured value is $\Delta \gamma=$ $0.018 \pm 0.001 \mathrm{~s}^{-1}$ (Fig. 4). The quantitative agreement of measurement and theory is rather remarkable, as the simple model presented above does not explicitly account for the finite temperature of the atoms, lattice trap anharmonicity, and the spatial variation of the lattice laser intensity giving rise to a spread in $\omega_{a t}$. These effects are only implicitly included in the measured $\gamma_{a t}$.

\section{Spread of atomic trap frequencies}

In a more elaborate model we consider the finite temperature of the atoms and the transverse spread in the trap frequencies originating from the Gaussian intensity profile of the lattice laser beam. We describe the atoms by a thermal density distribution $n(\mathbf{r})$ of constant $T=100 \mu \mathrm{K}$ in the lattice potential. For each atom in the distribution, we calculate $\omega_{a t}(\mathbf{r})$ from $V_{0}(\mathbf{r})$ and determine the corresponding membrane damping rate as in Eq. (5), but with $N=1$. We set $\gamma_{a t}=\gamma_{c}$, as the effects contributing to $\gamma_{\phi}$ are now explicitly modeled. We then sum the damping rates of all the atoms in the ensemble.

For the red theory curve in Fig. 3, we use a laser cooling rate of $\gamma_{c}=2 \pi \times 30 \mathrm{kHz}$. This is motivated by an estimate of the sub-Doppler laser cooling rate $\gamma_{c} \simeq \omega_{r}\left(2\left|\Delta_{\mathrm{MOT}}\right| / \Gamma_{s p}\right)$, where $\Gamma_{s p}=2 \pi \times 6.1 \mathrm{MHz}$ is the natural linewidth and $\omega_{r}=2 \pi \times 3.8 \mathrm{kHz}$ the recoil frequency of the ${ }^{87} \mathrm{Rb}$ cooling transition [40]. The MOT detuning of $\Delta_{\mathrm{MOT}}=-2 \pi \times 28 \mathrm{MHz}$ includes the light shift of the cooling transition due to the lattice laser. Notably, a change of $\pm 2 \pi \times 10 \mathrm{kHz}$ in $\gamma_{c}$ does not significantly change the shape 


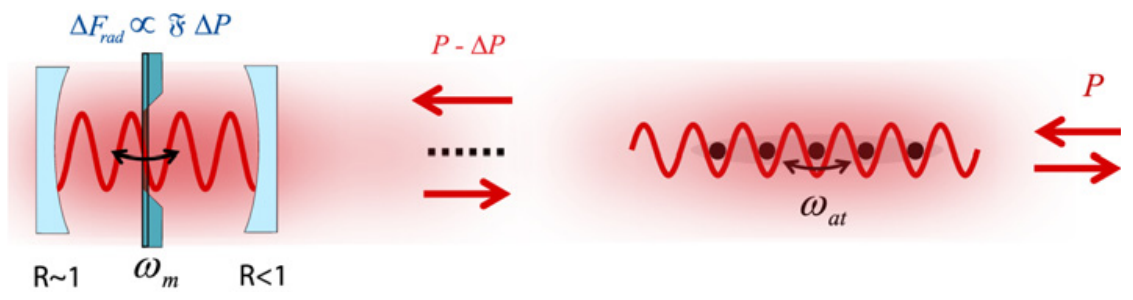

Figure 5. By placing a cavity around the membrane, the optomechanical coupling can be increased in proportion to the finesse $\mathfrak{F}$ of the cavity. Advantageously, this scheme does not require the atoms to be placed inside the same cavity, preserving the modularity of the system.

or magnitude of the theory curve in Fig. 3 of the main text. This analysis shows that the dephasing dominates $\gamma_{a t}$. The resulting line in Fig. 3 shows good agreement with the data for $w_{0}=370 \mu \mathrm{m}$ and $N=2.0 \times 10^{6}$, within the uncertainty of these parameters. For simplicity, $N$ is assumed to be constant for all power levels of the lattice laser beam across the resonance.

\section{CONCLUSIONS AND OUTLOOK}

We have realized a hybrid optomechanical system composed of ultracold atoms and a membrane where an optical lattice mediates a long-distance coupling. Despite the enormous mass difference between the atoms and the membrane, $N m / M \simeq 10^{-8}$, we clearly observe the backaction of the atoms onto the membrane. The measured change in the damping of the membrane and the predictions of the theoretical description in Ref. [23] agree remarkably well, suggesting that the theory can be used for extrapolation to optimized parameters.

The experiments presented in this article were performed with a setup where the optical access to the MOT chamber was rather limited, and we loaded only $2 \times 10^{6}$ atoms into the red detuned 1D optical lattice. In a dedicated setup, Raman sideband cooling could be used to prepare up to $3 \times 10^{8}$ atoms in the ground state of a large volume 3D lattice [38]. In this case, contributions to $\gamma_{a t}$ from spatial inhomogeneities and finite $T$ of the atoms would be much smaller. A blue-detuned lattice could be used to couple the atoms to the membrane. This would suppress effects due to spontaneous emission of the atoms and thus allow for smaller laser detuning and power. In the transverse direction the atoms could be confined by a far-detuned 2D lattice. In such a setup, the normal-mode splitting of the coupled atom-membrane system could be observed.

\section{Extensions of our setup}

The modularity of our setup allows one to easily modify either the atom or the membrane part. One promising modification is to place the membrane inside a cavity of finesse $\mathfrak{F}$, as illustrated in Fig. 5. In such a configuration the phase shift of the lattice due to the membrane displacement will be enhanced by $\mathfrak{F}$. As a result, the force acting onto the atoms will also increase by $\mathfrak{F}$. On the other hand, the cavity will enhance the power modulation of the lattice beam due to the atomic motion, resulting in a radiation pressure force on the membrane increased by $\mathfrak{F}$. Overall, these effects will lead to an increase of the atom-membrane coupling constant $g$ by the cavity finesse. Since the additional dissipation rate of the membrane $\Delta \gamma \propto g^{2}$, see Eq. (6), the sympathetic cooling performance will be increased by $\mathfrak{F}^{2}$. Hence, even a low finesse cavity can significantly enhance the sympathetic cooling rate. This scaling is valid until the strong coupling regime is reached, where $\Delta \gamma \sim g \propto \mathfrak{F}$. Furthermore, by making the cavity asymmetric such that the back mirror has a much higher reflectivity, almost all the power circulating in the cavity can be made to return to the atoms. 
An interesting feature of the proposed setup is that unlike in cavity-optomechanical cooling [39], the sideband-resolved regime is not required to achieve ground state cooling of the membrane. This is advantageous since this regime is experimentally challenging to reach for low-frequency oscillators such as the membranes. The corresponding condition in our setup is that the atoms can be ground-state cooled, which is routinely achieved in experiments [38]. Sympathetic cooling with atoms could also prove useful in the context of cooling other dielectric objects in a cavity such as levitated dielectric particles, which also have frequencies in the hundreds of $\mathrm{kHz}$ regime [41, 42].

In conclusion, we have realized an optomechanical interface between atoms and membrane. In this system, we have observed the back-action of atoms onto the membrane, which is prerequisite for cooling and manipulating the membrane with atoms. By implementing the improvements presented in the conclusion and outlook, sympathetic ground-state cooling of the membrane appears feasible.

We acknowledge our long-standing collaboration with the theory group of P. Zoller, in particular K. Hammerer, C. Genes, K. Stannigel, and M. Wallquist, as well as discussions with M. Ludwig and F. Marquardt. This work has been supported by the Nanosystems Initiative Munich (NIM), the EU project AQUTE, and the NCCR Quantum Science and Technology.

\section{References}

[1] S. Chu, Nature 416 (2002) 206.

[2] S. Chu, Science 253 (1991) 861.

[3] T. J. Kippenberg, K. Vahala, Opt. Exp. 15 (2007) 17172.

[4] T. J. Kippenberg, K. Vahala, Science 321 (2008) 1172.

[5] F. Marquardt, S. M. Girvin, Physics 2 (2009) 40.

[6] I. Favero, K. Karrai, Nature Photon. 3 (2009) 201.

[7] M. Aspelmeyer, P. Meystre, and K. Schwab, Phys. Today 65 (7), (2012) 29.

[8] J. Chan, T. P. M. Alegre, A. H. Safavi-Naeini, J. T. Hill, A. Krause, S. Gröblacher, M. Aspelmeyer, O. Painter, Nature 478 (2011) 89.

[9] S. Groeblacher, K. Hammerer, M. R. Vanner, M. Aspelmeyer, Nature 460 (2009) 724.

[10] E. Verhagen, S. Deleglise, S. Weis, A. Schliesser, T. J. Kippenberg, Nature 482 (2012) 63.

[11] J. D. Thompson, B. M. Zwickl, A. M. Jayich, F. Marquardt, S. M. Girvin, J. G. E. Harris, Nature 452 (2008) 72.

[12] W. Marshall, C. Simon, R. Penrose, D. Bouwmeester, Phys. Rev. Lett. 91 (2003) 130401.

[13] I. Pikovski, M. R. Vanner, M. Aspelmeyer, M. S. Kim, C. Brukner, Nature Physics 8 (2012) 393.

[14] D. E. McClelland, N. Mavalvala, Y. Chen, and R. Schnabel, Laser \& Photonics Reviews 5 (2011) 677.

[15] D. Meiser, P. Meystre, Phys. Rev. A 73 (2006) 033417.

[16] C. Genes, D. Vitali, P. Tombesi, Phys. Rev. A 77 (2008) 050307.

[17] H. Ian, Z. R. Gong, Y. x. Liu, C. P. Sun, F. Nori, Phys. Rev. A 78 (2008) 013824.

[18] Y. Chang, T. Shi, Y. x. Liu, C. P. Sun, F. Nori, Phys. Rev. A 83 (2011) 063826.

[19] A. B. Bhattacherjee, Phys. Rev. A 80 (2009) 043607.

[20] K. Hammerer, M. Aspelmeyer, E. S. Polzik, P. Zoller, Phys. Rev. Lett. 102 (2009) 020501.

[21] K. Hammerer, M. Wallquist, C. Genes, M. Ludwig, F. Marquardt, P. Treutlein, P. Zoller, J. Ye, H. J. Kimble, Phys. Rev. Lett. 103 (2009) 063005.

[22] M. Wallquist, K. Hammerer, P. Zoller, C. Genes, M. Ludwig, F. Marquardt, P. Treutlein, J. Ye, H. J. Kimble, Phys. Rev. A 81 (2010) 023816.

[23] K. Hammerer, K. Stannigel, C. Genes, P. Zoller, P. Treutlein, S. Camerer, D. Hunger, T. W. Hänsch, Phys. Rev. A 82 (2010) 021803. 
[24] D. Hunger, S. Camerer, M. Korppi, A. Jöckel, T. Hänsch, P. Treutlein, Comptes Rendus Physique 12 (2011) 871

[25] Y. J. Wang, M. Eardley, S. Knappe, J. Moreland, L. Hollberg, J. Kitching, Phys. Rev. Lett. 97 (2006) 227602.

[26] D. Hunger, S. Camerer, T. W. Hänsch, D. König, J. P. Kotthaus, J. Reichel, P. Treutlein, Phys. Rev. Lett. 104 (2010) 143002.

[27] S. Camerer, M. Korppi, A. Jöckel, D. Hunger, T. W. Hänsch, P. Treutlein, Phys. Rev. Lett. 107 (2011) 223001.

[28] B. M. Zwickl, W. E. Shanks, A. M. Jayich, C. Yang, A. C. B. Jayich, J. D. Thompson, J. G. E. Harris, Appl. Phys. Lett. 92 (2008) 103125.

[29] D. J. Wilson, C. A. Regal, S. B. Papp, and H. J. Kimble, Phys. Rev. Lett. 103 (2009) 207204.

[30] A. Jöckel, M. T. Rakher, M. Korppi, S. Camerer, D. Hunger, M. Mader, P. Treutlein, Appl. Phys. Lett. 99 (2011) 143109.

[31] M. Karuza, C. Biancofiore, C. Molinelli, M. Galassi, R. Natali, P. Tombesi, G. Di Giuseppe, D. Vitali, ArXiv e-prints (2012), 1209.1352.

[32] D. E. Chang, K. K. Ni, O. Painter, H. J. Kimble, New Journal of Physics 14 (2012) 045002.

[33] J. Reichel, W. Hänsel, T. W. Hänsch, Phys. Rev. Lett. 83 (1999) 3398.

[34] I. Bloch, Nature Phys. 1 (2005) 23.

[35] R. Grimm, M. Weidemüller, Y. Ovchinnikov, Adv. At. Mol. Opt. Phys. 42 (2000) 95.

[36] G. Raithel, W. D. Phillips, S. L. Rolston, Phys. Rev. Lett. 81 (1998) 3615.

[37] A. Görlitz, M. Weidemüller, T.W. Hänsch, A. Hemmerich, Phys. Rev. Lett. 78 (1997) 2096.

[38] A. J. Kerman, V. Vuletic, C. Chin, S. Chu, Phys. Rev. Lett. 84 (2000) 439.

[39] F. Marquardt, J. P. Chen, A. A. Clerk, S. M. Girvin, Phys. Rev. Lett. 99 (2007) 093902.

[40] H. J. Metcalf, P. van der Straten, Laser Cooling and Trapping (Graduate Texts in Contemporary Physics) (Springer, 2001), ISBN 0387987282.

[41] O. Romero-Isart, M. L. Juan, R. Quidant, J. I. Cirac, New Journal of Physics 12 (2010) 033015.

[42] D. E. Chang, C. A. Regal, S. B. Papp, D. J. Wilson, J. Ye, O. Painter, H. J. Kimble, P. Zoller, PNAS 107 (2010) 1005. 